\title{
Public Health Surveillance of Habitual Physical Activity in Adolescents and Adults in Namibia: A Cross-sectional Validation of Activity Questionnaires against Accelerometry
}

Authors: Hilde L Nashandi ${ }^{1^{*}}$, John J Reilly ${ }^{2}$, Xanne Janssen ${ }^{2}$

1 University of Namibia, Faculty of Health Sciences, School of Nursing, Windhoek, Namibia

2 University of Strathclyde Glasgow, Physical Activity for Health Group, Glasgow, Scotland G1 1XQ

*Address for correspondence

H.L. Nashandi, MPH, School of Nursing, Faculty of Health Sciences, University of Namibia, Private Bag 13301, 340 Mandume Ndemufayo Ave, Pionierspark, Windhoek, NAMIBIA. Email:

hildeliisa@yahoo.com

Keywords: Physical activity; Global Physical Activity Questionnaire; validity; accelerometer; obesity; exercise; Africa

This is a peer-reviewed, author accepted manuscript of the following research article: Nashandi, H. L., Reilly, J. J., \& Janssen, X. (2020). Public health surveillance of physical activity in adolescents and adults in Namibia: a cross-sectional validation of activity questionnaires against accelerometry. Journal of Public Health. https://doi.org/10.1093/pubmed/fdaa067 


\section{ABSTRACT}

Background: Monitoring population-level physical activity is crucial for examining adherence to global guidelines and addressing obesity. This study validated self-reported moderate-to-vigorous physical activity (MVPA) against an accurate device-based method in Namibia.

Methods: Adolescent girls ( $\mathrm{n} 52$, mean age 16.2y [SD 1.6]) and adult women ( $\mathrm{n} 51$, mean age 31.3y [SD 4.7]) completed the PACE+/GPAQ self-report questionnaires, and were asked to wear an Actigraph accelerometer for seven days. Validity of self-reported MVPA was assessed using rank order correlations between self-report and accelerometry, and classification ability of the questionnaires with Mann-Whitney tests, kappa's, sensitivity and specificity.

Results: In the adolescents, Spearman's rank coefficients between self-reported MVPA (days/week) and accelerometry measured MVPA were positive but not significant $(r=0.240, p=0.104)$. In the adults, self-reported MVPA (minutes/day) was moderately and significantly correlated with accelerometermeasured MVPA ( $r=0.396, p=0.008)$. In both groups there was fair agreement between accelerometry and questionnaire-defined tertiles of MVPA (adolescents $k=0.267 ; p=0.010$; adults $k=0.284 ; p=0.008$ ), and measured MVPA was significantly higher in the individuals self-reporting higher MVPA than those reporting lower MVPA.

Conclusions: The PACE+ and GPAQ questionnaires have a degree of validity in adolescent girls and adult females in Namibia, though more suitable for population than individual level measurement.

Keywords: Physical activity, Validity, Adult women, Adolescent girls

This is a peer-reviewed, author accepted manuscript of the following research article: Nashandi, H. L., Reilly, J. J., \& Janssen, X. (2020). Public health surveillance of physical activity in adolescents and adults in Namibia: a cross-sectional validation of activity questionnaires against accelerometry. Journal of Public Health. https://doi.org/10.1093/pubmed/fdaa067 
This is a peer-reviewed, author accepted manuscript of the following research article: Nashandi, H. L., Reilly, J. J., \& Janssen, X. (2020). Public health surveillance of physical activity in adolescents and adults in Namibia: a cross-sectional validation of activity questionnaires against accelerometry. Journal of Public Health. https://doi.org/10.1093/pubmed/fdaa067 


\section{INTRODUCTION}

There has been a "physical activity transition"1’2 across Africa: levels of moderate-to-vigorous physical activity (MVPA) are now very low ${ }^{3 \cdot 2 \cdot 4}$ particularly among adolescent girls and adult women, who are also at highest risk of obesity across Sub-Saharan Africa ${ }^{3 \cdot 5}$. In Namibia, childhood obesity prevalence is high and probably increasing ${ }^{6}$. The 2013 Global School-based Student Health Survey (GSHS) reported that in Namibia, $10.4 \%$ of females aged 13-17 were overweight or obese, and the prevalence of insufficient physical activity among adolescents exceeded $80 \%{ }^{4}$. In Namibia, only $5 \%$ of women and $12 \%$ of men age $15-49$ are physically active at work, while only $16 \%$ of women and $32 \%$ percent of men engage in non-work-related physical activity ${ }^{7}$.

Surveillance of habitual MVPA levels in adolescents and adults in most countries still depends on subjective, self-report, measures. For example, the $\mathrm{PACE}+{ }^{8}$ is used widely among adolescents as part of the Global Student Health Survey (https://www.cdc.gov/GSHS/), and the General Physical Activity Questionnaire (GPAQ) $)^{9}$ is used widely as part of the WHO 'STEPS' global NCD surveillance (https://www.who.int/ncds/surveillance/steps/en/). However, the validity of MVPA measured by selfreport is in question-in children and adolescents few self-report measures have been subject to validations against more accurate measures of MVPA such as accelerometry ${ }^{10}$. In the adolescent population, there is a dearth of validation study evidence from low-middle income countries ${ }^{11}$ and some validations from high-income countries have been discouraging ${ }^{12}$. Out of 38 validation studies included in a recent review, only two showed acceptable correlations ( $r>0.40)$ compared to accelerometry $^{13}$. In adults too, validation of many self-report questionnaires used in public health surveillance has been rare in low-middle income countries, and in high-income countries results have also been discouraging. For example, prevalence of meeting physical activity guidelines may be overestimated by self-report in adults in the UK and USA ${ }^{14,15}$. A recent review of physical activity measures showed the mean difference between questionnaires using a 7-day recall (like those included in this study) and the gold standard (doubly labelled water) ranged from an underestimation of $60 \%$ to an overestimation of $60 \%{ }^{16}$.

Increasing habitual physical activity among children, adolescents, and adults is central to prevention of obesity and many non-communicable diseases (NCDs) $)^{17,18,19}$. If global targets are to be met, or even monitored, unbiased methods of public health surveillance of physical activity are required. The This is a peer-reviewed, author accepted manuscript of the following research article: Nashandi, H. L., Reilly, J. J., \& Janssen, X. (2020). Public health surveillance of physical activity in adolescents and adults in Namibia: a cross-sectional validation of activity questionnaires against accelerometry. Journal of Public Health. https://doi.org/10.1093/pubmed/fdaa067 
primary aim of the present study was therefore to validate the self-report of habitual MVPA among adolescent girls (using the PACE+ questionnaire) and among adult women (using the GPAQ) against an accurate device-based method (accelerometry) in Namibia.

\section{METHODS}

\section{Study sample, recruitment and ethics}

The project was approved by the Ministry of Health and Social Services Research Management Committee. All participants provided informed written consent. The present validation study was part of a larger study focusing on lifestyle and obesity risk among adolescent girls and adult women in Namibia. Participants of this larger study were drawn from urban public schools in Khomas region using stratified random sampling of schools. For the larger study a total of 207 adolescent girls' participants had their parents give consent and 212 women provided consent and participated in the study. A subsample of 52 adolescents and 51 adult women were randomly selected (using a random number selector) to take part in the current study. All measures were taken at school during school time. In addition, questionnaires regarding physical activity were completed via interviews at schools. Body fat percentage was measured accurately via deuterium oxide dilution method, as described in detail elsewhere ${ }^{6,20}$ though this variable was used only for descriptive purposes in the present study.

\section{Measurement of habitual MVPA by accelerometry}

Participating (n 52) adolescents and adults ( $\mathrm{n}$ 51) were given an Actigraph GT3X+ accelerometer (ActiGraph, Pensacola, FL) to wear on the right hip for 7 full days, taking the device off only when sleeping or during water based activities, as described previously ${ }^{21,22}$. The Actigraph monitor is an acceptable criterion measure for evaluating questionnaire validity ${ }^{9}$. For feasibility reasons and to reduce participant burden, accelerometers were distributed to participants on the same day as questionnaire completion. Data was collected in $30 \mathrm{~Hz}$ and transformed into 15 -second epochs. To be included in the analysis participants required at least 3 valid days (i.e., at least 10 hours of wear), including at least one weekend day, in order to obtain an estimate of habitual MVPA ${ }^{23,24,25}$. Data from the devices were downloaded and cleaned using Actilife software version 6.12.1 (ActiGraph, Pensacola, FL). Non-wear time was removed using a 60 minutes of consecutive zeros criteria ${ }^{21}$. The Actilife data scoring programme was used to determine daily minutes spent in sedentary time, light PA intensity (LPA), moderate PA intensity (MPA), vigorous PA intensity (VPA), and MVPA using the Evenson activity cut point for adolescents which is most accurate to use in this age group ${ }^{26}$ and the This is a peer-reviewed, author accepted manuscript of the following research article: Nashandi, H. L., Reilly, J. J., \& Janssen, X. (2020). Public health surveillance of physical activity in adolescents and adults in Namibia: a cross-sectional validation of activity questionnaires against accelerometry. Journal of Public Health. https://doi.org/10.1093/pubmed/fdaa067 
Freedson activity cut point for adults ${ }^{27}$. Both cut points have good evidence of validity in adolescents and adults respectively.

\section{Measurement of habitual MVPA by self-report}

In the adolescent sample, self-reported MVPA was obtained using the PACE+ questionnaire completed at school. The PACE+ is used widely as a self-report measure of habitual MVPA in adolescents and has some evidence of validity in western, high-income, settings ${ }^{28}$ as well as satisfactory reliability ${ }^{29,30}$ This questionnaire asks study participants to report the number of days in the last week when they engaged in MVPA for at least 60 minutes (examples of activities are provided, MVPA is explained). Additionally, students reported the following outcomes: VPA during leisure time (number of days per week and hours per week); mode of transport to and from school per day; time spent walking to school (hours/day); time spent watching TV (hours/day); and time spent playing games on phone or computer (hours/day). The questionnaire was completed via an interview in English or the participants' local language on the first day of accelerometer wear.

In the adult sample, the GPAQ ${ }^{9}$ questionnaire was used to estimate habitual MVPA (minutes/day and minutes/week). GPAQ is widely used globally for surveillance of compliance with physical activity levels in adults as part of the WHO STEPS NCD surveillance programme, and also provides a measure of sitting time ${ }^{9,31}$. The GPAQ has shown moderate reliability and poor to fair criterion validity ${ }^{9,32}$. The questionnaire was completed via an interview in English or the participants' local language on the first day of accelerometer wear.

\section{Other measures}

Two trained researchers measured height to $0.1 \mathrm{~cm}$ and weight to $0.1 \mathrm{~kg}$ using standard methods ${ }^{33}$. Weight was measured using electronic SECA scale and the participant were asked to remove their footwear and socks; to have light clothes on and no items in the pockets. BMI was calculated as $\mathrm{kg} / \mathrm{m}^{2}$ and in the adults obesity was defined as $\mathrm{BMI}>30.0 \mathrm{~kg} / \mathrm{m}^{2}$; in the adolescents BMI-for-age $Z$ score was calculated using WHO reference data and obesity was defined as BMI-for-age $Z$ score $\geq+2.00$ SD $^{33}$.

\section{Statistical analyses}

Validity of each questionnaire was assessed in three ways. First, we determined the ability of the questionnaire to rank order MVPA as measured by accelerometry, using Spearman's rank-order correlation. Correlations were classed as: 0.00-0.19 'very weak'; 0.20-0.39 'weak'; 0.40-0.59 This is a peer-reviewed, author accepted manuscript of the following research article: Nashandi, H. L., Reilly, J. J., \& Janssen, X. (2020). Public health surveillance of physical activity in adolescents and adults in Namibia: a cross-sectional validation of activity questionnaires against accelerometry. Journal of Public Health. https://doi.org/10.1093/pubmed/fdaa067 
'moderate', 0.60-0.79 'strong'; 0.8-1.0 'very strong'. Additionally, we examined the differences in MVPA between those meeting the physical activity guidelines and those who did not using a MannWhitney $U$ test. As a third measure of validity of self-report participants were rank ordered from highest-lowest MVPA by accelerometry and divided into tertiles. We then assessed the ability of the PACE+ and GPAQ to place individuals in thirds of MVPA by accelerometry using kappa statistics with the standard descriptors for kappa by Landis and Koch : $0.21-0.40$ 'fair agreement'; $0.41-0.60$ 'moderate agreement'; 0.61-0.80 'substantial agreement'; and 0.81-1.00 'almost perfect agreement ${ }^{\prime 34}$. We also assessed the ability of the PACE+ and GPAQ to classify individuals as meeting or not meeting the physical activity guidelines by reporting percentage agreement, sensitivity and specificity. Data was dichotomized by categorizing participants in meeting the guidelines versus not meeting the guidelines on $\geq 3$ days versus $<3$ days and $\geq 5$ days versus $<5$ days for adolescents, and $\geq 150$ minutes/week versus $<150$ minutes/week for the adults. As adherence with the MVPA guidelines in adolescents is often reported using an average MVPA/day, the average MVPA/day was also calculated and data was dichotomised using a $\geq 60 \mathrm{~min}$ of MVPA/day versus $<60$ minutes/day criteria ${ }^{35}$.

Power of the analyses was difficult to assess at the outset and was fixed by the size of the present study in which subsamples of a much larger study were recruited for the validations reported here. The sample size in the present study was similar to or larger than many previous validations of selfreported physical activity against accelerometry ${ }^{10}$.

\section{RESULTS}

\section{Characteristics of study participants}

Of the 52 adolescents and 51 adults invited to take part in the accelerometry protocol 47 adolescents $(90 \%)$ and 44 adults (86\%) provided the 3 days of accelerometry required for measurement of habitual PA. The mean wear time was 5.4 days (SD 1.3) and 14.4 hours/day (1.6) in the adolescents and 5.2 days (1.6) and 15.0 hours/day (2.3) in the adults.

In both the adolescent sample and adult sample no significant differences in age, BMI and fat mass were found between those who completed the accelerometry and those who did not. Participant characteristics are given in Table 1.

A total of $3(6.4 \%)$ adolescents reported meeting the MVPA guideline on 7 days per week, $6(12.8 \%)$ reported meeting this on at least 5 days per week, and 17 (36.1\%) reported meeting it on at least 3

This is a peer-reviewed, author accepted manuscript of the following research article: Nashandi, H. L., Reilly, J. J., \& Janssen, X. (2020). Public health surveillance of physical activity in adolescents and adults in Namibia: a cross-sectional validation of activity questionnaires against accelerometry. Journal of Public Health. https://doi.org/10.1093/pubmed/fdaa067 
days per week. Levels of MVPA by accelerometry among the adolescents averaged 36.8 (SD 22.2) minutes/day.

In the adults, mean self-reported daily MVPA was 40.6 minutes/day (70.5), and 13 (27.7\%) adults reported an average of $\geq 150$ minutes/week in MVPA. Levels of MVPA measured by accelerometry in the adults averaged 30.2 minutes/day (22.1).

\section{Rank order correlation between self-reported and measured MVPA}

In the adolescents, Spearman's rank order correlation coefficients between self-reported MVPA (days/week) and accelerometry measured MVPA (days/week) were weak and not significant ( $r=0.240$ $\mathrm{p}=0.104$ ). In the adults, self-reported MVPA (minutes/day) was moderately and significantly correlated with accelerometer-measured MVPA (minutes/day; $r=0.396, P=0.008$ ).

\section{Classification ability of self-report methods}

Adolescent participants meeting the guidelines on three days per week based on self-reported MVPA engaged on average in an extra $20.7 \mathrm{~min} \mathrm{MVPA}$ /day (SD $6.9 \mathrm{~min} /$ day) as measured by accelerometry compared to those meeting the guidelines on less than three days per week $(p=0.005)$. Adult participants meeting the guidelines based on self-report spent on average $14.1 \mathrm{~min} / \mathrm{day}$ (SD 7.1 min/day) more time in MVPA as measured by accelerometry compared to those who reported they did not meet the guidelines $(p=0.053)$.

Classification accuracy is shown in Table 2. In the adolescents there was fair agreement between accelerometry defined tertiles of MVPA and self-reported tertiles MVPA ( $k=0.267 ; p=0.010)$. In addition, there was fair agreement between accelerometry defined and self-reported adherence to the $60 \mathrm{~min} /$ day MVPA guideline based on meeting the guideline on at least 3 days/week $(\mathrm{k}=0.261$; $p=0.035$ ). The PACE showed a sensitivity of $71.4 \%$ and a specificity of $70.0 \%$ for meeting 60 min of MVPA/day on an average of 3 or more days.

In the adults there was fair agreement between accelerometry defined tertiles of MVPA and selfreported tertiles MVPA $(k=0.284 ; p=0.008)$. In addition, there was fair agreement between accelerometry defined and self-reported adherence to the $150 \mathrm{~min} /$ week MVPA guideline $(\mathrm{k}=0.225$; $p=0.081$ ). The GPAQ showed a sensitivity of $40 \%$ and a specificity of $84.2 \%$ for guideline adherence classification.

This is a peer-reviewed, author accepted manuscript of the following research article: Nashandi, H. L., Reilly, J. J., \& Janssen, X. (2020). Public health surveillance of physical activity in adolescents and adults in Namibia: a cross-sectional validation of activity questionnaires against accelerometry. Journal of Public Health. https://doi.org/10.1093/pubmed/fdaa067 


\section{DISCUSSION}

\section{Main findings of this study}

The present study found that, for both the adolescent girls and the adult women, there was a degree of consistency between self-reported and accelerometry measured MVPA. The questionnaires performed slightly better in the adult women than the adolescent girls. All of the tests of association or agreement were positive and most were statistically significant.

\section{What is already known on this topic}

The School-based Students Survey in Namibia found that the prevalence of not-meeting habitual MVPA guidance (for those aged $11-17$ years) was $87.4 \%^{36}$, suggesting that the relatively low MVPA observed in the adolescents in the present study (well below the recommended minimum of 60 minutes MVPA per day) was not unusual. On average, the adult women in our sample met the international MVPA guidelines as measured by accelerometry.

A previous questionnaire-based survey in Namibia also found that adolescent MVPA was low ${ }^{36}$. Few direct comparisons of the present study with other studies are available because of the dearth of similar studies in Sub-Saharan Africa. However the results of a recent study which validated the GPAQ in Bangladeshi adult women ${ }^{9}$ found similar results to the current study. More validation evidence from high-income countries is available ${ }^{37}$. In the current study, PACE showed a sensitivity of $71.4 \%$ and a specificity of $70 \%$ for meeting $60 \mathrm{~min}$ of MVPA/day on 3 or more days. This is in line with another study.

Similar results were found in the adult sample, with GPAQ sensitivity $40 \%$ and specificity $84 \%$ for guideline adherence. The moderate correlations between self-reported MVPA and accelerometry MVPA in adults in the present study is in agreement with results reported in a systemic review of mainly European studies where correlations between questionnaire and accelerometer ranged from $r=-0.02$ to $r=0.49(P<0.01)$ for adult women. Another study also found generally weak to moderate correlations ( $r=-0.02$ to $r=0.79$ ) between the different approaches ${ }^{38}$. In summary, the lowmoderate positive correlations and fair agreement between questionnaires and accelerometry in the present study were consistent with studies in European populations of adolescents and adults. The similarity in validation results between the present study and those obtained from similar studies in 
western, high-income, countries, was not expected, since self-report questionnaires of MVPA developed in high-income countries may lack validity in non-western countries due to cultural differences between populations ${ }^{2,4}$.

\section{What this study adds}

The present study suggests that MVPA from the widely used and recommended questionnaires in adolescents (PACE+, used in the GSHS globally) and adults (GPAQ, used in the WHO STEPS surveillance programmes globally) is related to habitual MVPA from accelerometry. In some countries measures of MVPA by questionnaire in national surveys bear no relation to measured MVPA by accelerometer ${ }^{12}$, and there is a concern that validity of many physical activity questionnaires which are considered standard in high-income western countries might be less appropriate in other cultures. While the present study is broadly supportive of these questionnaires, it is worth noting that tests of association were strong, and there was a good deal of disagreement between MVPA from the questionnaires versus the accelerometer on an individual level. In summary, these questionnaires might be best restricted to national surveillance of habitual MVPA, and not assessment of individual MVPA. Although use of accelerometry remains the preferred option when measuring levels of physical activity, this is not always feasible. Using accelerometry requires a large amount of resources and expertise, which is currently lacking in many countries. The use of questionnaires is more feasible and therefore the findings of the current study are promising and enable low-income countries to collect surveillance data on a population wide level.

\section{Limitations of this study}

The present study had several strengths. Firstly, novelty: this was the first study from Southern Africa which validated these widely used questionnaires in adolescent girls and adult women, the two groups in the population most critical to the African obesity epidemic.${ }^{17}$ Second, the present study was a test of criterion validity since it used accelerometry. The present study also had a number of weaknesses. Results cannot be generalised with confidence to adolescent boys or adult men, or to other environments (e.g. rural areas). Further studies will be needed to assess generalisability. In addition, sample size was too small to provide adequate power for analysis of factors which influenced questionnaire accuracy. The questionnaire was also administered as an interview- this may have influenced validity and return rates. Interview-based questionnaires are more expensive than postal This is a peer-reviewed, author accepted manuscript of the following research article: Nashandi, H. L., Reilly, J. J., \& Janssen, X. (2020). Public health surveillance of physical activity in adolescents and adults in Namibia: a cross-sectional validation of activity questionnaires against accelerometry. Journal of Public Health. https://doi.org/10.1093/pubmed/fdaa067 
questionnaires, but are already used widely in NCD surveillance, e.g. the WHO STEPS Survey is interviewer based. Last, the questionnaire was completed on the first day of accelerometer wear, this may have influenced the results given the questionnaire asks about participants activity during the last 7 days (i.e. before the accelerometer measure took place). However, both accelerometer and questionnaire measures should provide habitual levels of physical activity and so this should not result in marked differences between methods. In addition, the PACE+ questionnaire was validated following the same approach ${ }^{8}$. Last, it is worth noting that adult accelerometry data was analysed using 15seconds epochs. It is currently unknown what the most optimal epoch setting is for adults ${ }^{39}$, however, child and adolescent literature shows that shorter epochs provide more valid MVPA measures.

\section{CONCLUSION}

This study shows a degree of validity of the most widely used public health surveillance instruments, the PACE+ and GPAQ for group/population measures of MVPA. Their use could enhance surveillance, identifying trends and population levels of physical activity in Namibia.

\section{REFERENCES}

1 Muthuri, S.K., Francis, C.E., Wachira, L.M., LeBlanc, A. G., Sampson, M., Vincent O. Onywera, V.O., \& Tremblay, M.S. (2014). Evidence of an Overweight/Obesity Transition among School-Aged Children and Youth in Sub-Saharan Africa: A Systematic Review. The PLOS ONE.

2 Tremblay, M.S., Longmuir, P.E., Barnes, J.D. et al. (2018). Physical literacy levels of Canadian children aged 8-12 years: descriptive and normative results from the RBC Learn to Play-CAPL project. BMC Public Health 18, 1036 (2018) doi: 10.1186/s12889-018-5891-x.

3 Craig, E., Reilly, J.J., \& Bland, R. (2016). Risk factors for overweight and overfatness in rural South African children and adolescents. Journal of Public Health | Vol. 38, No. 1, pp. 24-33 | doi:10.1093/pubmed/fdv016 | Advance Access Publication March 4, 2015.

4 Guthold, R., Stevens, G.A., Riley, L.M., \& Bull, F. C. (2019). Global trends in insufficient physical activity among adolescents: a pooled analysis of 298 population-based surveys with 1.6 million participants. Lancet Child Adolesc Health 2019. Published Online November 21, 2019 https://doi.org/10.1016/S2352-4642(19)30323-2

5 Pradeilles, R., Griffiths, P. L., Norris, S.A., Feeley, A.B. \& Rousham, E.K. (2015). Socio-economic influences on anthropometric status in urban South African adolescents: sex differences in the Birth to Twenty Plus cohort. Public Health Nutrition, 18(16), pp.1998-3012.

This is a peer-reviewed, author accepted manuscript of the following research article: Nashandi, H. L., Reilly, J. J., \& Janssen, X. (2020). Public health surveillance of physical activity in adolescents and adults in Namibia: a cross-sectional validation of activity questionnaires against accelerometry. Journal of Public Health. https://doi.org/10.1093/pubmed/fdaa067 
6 Diouf, A, Adom, T., Aouidet, A., et al. Body mass index vs deuterium dilution method for establishing childhood obesity prevalence, Ghana, Kenya, Mauritius, Morocco, Namibia, Senegal, Tunisia and United Republic of Tanzania. Bulletin World Health Organisation. 2018; 96:772-781

7 Ministry of health and Social Services.2015. Namibia Demographic and Health Survey 2013. Windhoek, Namibia.

8 Murphy, M. H., Rowe, D. A., Belton, S., \& Woods, C. B. Validity of a two-item physical activity questionnaire for assessing attainment of physical activity guidelines in youth. BMC Public Health, (2015). 15(1), 1080.

9 Mumu, S.J., Ali, L., Barnett, A., and Merom, D. Validity of the global physical activity questionnaire (GPAQ) in Bangladesh. BMC Public Health (2017) 17:650 DOI 10.1186/s12889-017-4666-0

10 Biddle, J.H., Gorely, T., Natalie Pearson, N., \& Bull, F.C. (2011). An assessment of self-reported physical activity instruments in young people for population surveillance: Project ALPHA Stuart. International Journal of Behavioral Nutrition and Physical Activity 2011, 8:1 http://www.ijbnpa.org/content/8/1/1

11 El Hamdouchi, A., Adom, T., Aouidet, A., et al. (2019). Protocol for validating simple measures of body fatness and physical activity of children in twelve African countries: The ROUND-IT Africa Study. African Journal for Physical Activity and Health Sciences, 25(2), 142-173.

12 Basterfield, L., Adamson, A.J., Parkinson, K.N., Maute, U., Li, P.X., and Reilly, J.J. (2008). Surveillance of physical activity in the UK is flawed: validation of the Health Survey for England Physical Activity Questionnaire. Arch. Dis. Child. 93(12): 1054-1058. doi:10.1136/adc.2007.135905.

13 Hidding, L. M., Chinapaw, M. J., van Poppel, M. N., Mokkink, L. B., \& Altenburg, T. M. (2018). An updated systematic review of childhood physical activity questionnaires. Sports Medicine, 48(12), 2797-2842.

14 Townsend, N., Bhatnagar, P., Wickramasinghe, K., Scarborough, P., Foster, C., \& Rayner, M. (2012). Physical activity statistics 2012. British Heart Foundation: London.

15 Gill, J.M.R., Celis-Morales, C.A., \& Ghouri, N. (2013). Review Physical activity, ethnicity and cardiometabolic health: Does one size fit all? http://dx.doi.org/10.1016/j.atherosclerosis.2013.11.039. www.elsevier.com/locate/atherosclerosis.

16 Dowd, K. P., Szeklicki, R., Minetto, M. A., Murphy, M. H., Polito, A., Ghigo, E., ... \& Tomczak, M. (2018). A systematic literature review of reviews on techniques for physical activity measurement in adults: a DEDIPAC study. International journal of behavioral nutrition and physical activity, 15(1), 15.

17 World Health Organisation. (2016). WHO Ending Childhood Obesity ('ECHO' Report) 2016. Geneva, Switzerland.

18 World Health Organisation. Global action plan on physical activity 2018-2030: more active people for a healthier world. Geneva: World Health Organization; 2018. Licence: CC BY-NC-SA 3.0 IGO.

19 World Health Organisation. (2017). 'Best buys' and other recommended interventions for the prevention and control of Noncommunicable diseases. Geneva, Switzerland.

This is a peer-reviewed, author accepted manuscript of the following research article: Nashandi, H. L., Reilly, J. J., \& Janssen, X. (2020). Public health surveillance of physical activity in adolescents and adults in Namibia: a cross-sectional validation of activity questionnaires against accelerometry. Journal of Public Health. https://doi.org/10.1093/pubmed/fdaa067 
20 International Atomic Energy Agency. (2010). IAEA Human Health Series No.12. Introduction to Body Composition assessment using the Deuterium dilution Technique with Analysis of Saliva Samples by Fourier Transform Infrared Spectrometry. Vienna, Austria.

21 Janssen, X., Basterfield, L., Parkinson, K. N., Pearce, M. S., Reilly, J. K., Adamson, A. J., ... \& Gateshead Millennium Study Core Team. (2015). Objective measurement of sedentary behavior: impact of nonwear time rules on changes in sedentary time. BMC public health, 15(1), 504.

22 Janssen, X., Basterfield, L., Parkinson, K.N. et al. Non-linear longitudinal associations between moderate-to-vigorous physical activity and adiposity across the adiposity distribution during childhood and adolescence: Gateshead Millennium Study. Int J Obes (2019): 43, 744-750

23 Cain, Kelli L., et al. "Using accelerometers in youth physical activity studies: a review of methods." Journal of Physical Activity and Health 10.3 (2013): 437-450.Kristense

24 Mattocks, C., et al. Use of accelerometers in a large field-based study of children: protocols, design issues, and effects on precision. Journal of Physical Activity and Health, 2008, 5(s1), S98-S111.

25 Kristensen, Peter Lund, et al. The association between aerobic fitness and physical activity in children and adolescents: the European youth heart study. European journal of applied physiology 110.2 (2010): 267-275.

26 Trost, S.G., Loprinzi, P.D., Moore, R.,\& Pfeiffer, K.A. Comparison of accelerometer cut points for predicting activity intensity in youth. Medicine and science in sports and exercise 43.7 (2011): 13601368.

27 Freedson P, Pober D, Janz KF. Calibration of accelerometer output for children. Med Sci Sports Exerc. 2005; 37 (11 Suppl):S523-30.

28 Prochaska, J.J., Sallis, J.F. and Long, B. (2001). A physical activity screening measure for use with adolescents in primary care. Archives of Pediatrics and Adolescent Medicine, 155(5): 554-559.

29 Bobakova, D., Hamrik, Z., Badura, P., Sigmundova, D., Nalecz, H., \& Kalman, M. (2015). Test-retest reliability of selected physical activity and sedentary behaviour HBSC items in the Czech Republic, Slovakia and Poland. International Journal of Public Health, 60(1), 59-67.

30 Liu, Y., Wang, M., Tynjälä, J., Lv, Y., Villberg, J., Zhang, Z., \& Kannas, L. (2010). Test-retest reliability of selected items of Health Behaviour in School-aged Children (HBSC) survey questionnaire in Beijing, China. BMC Medical Research Methodology, 10(1), 73.

31 World Health Organization. WHO STEPS surveillance manual: the WHO STEPwise approach to chronic disease risk factor surveillance. Geneva, Switzerland: World Health Organization; 2005.

32 Bull FC, Maslin TS, Armstrong T. (2009) Global physical activity questionnaire (GPAQ) nine country reliability and validity study. Journal of Physical Activity and Health, 6: 790-804.

33 World Health Organization. (2006). WHO Child Growth Standards: World Health Organization. Geneva

34 Landis JR, Koch GG. (1977) The measurement of observer agreement for categorical data. Biometrics, 33(1):159-74.

This is a peer-reviewed, author accepted manuscript of the following research article: Nashandi, H. L., Reilly, J. J., \& Janssen, X. (2020). Public health surveillance of physical activity in adolescents and adults in Namibia: a cross-sectional validation of activity questionnaires against accelerometry. Journal of Public Health. https://doi.org/10.1093/pubmed/fdaa067 
35 Ridgers, N. D., Timperio, A., Crawford, D., \& Salmon, J. (2012). Validity of a brief self-report instrument for assessing compliance with physical activity guidelines amongst adolescents. Journal of Science and Medicine in Sport, 15(2), 136-141.

36 Ministry of health and Social Services. 2008. Report on the Namibia School- Based Student Health Survey 2004. Windhoek, Namibia.

37 Skender, S., Ose, J., Chang-Claude, J. , Paskow, M., Brühmann,B., . Siegel, E. M., Steindorf, S., and. Ulrich, C.M. (2016). Accelerometry and physical activity questionnaires - a systematic review. BMC Public Health (2016) 16:515.

38 Kowalski, K., Rhodes, R., Naylor, P.J., Tuokko, H., \& MacDonald, S. Direct and indirect measurement of physical activity in older adults: a systematic review of the literature. International Journal of Behavioral Nutrition and Physical Activity 2012, 9:148

39 Migueles, Jairo $\mathrm{H}_{\text {., }}$ et al. Accelerometer data collection and processing criteria to assess physical activity and other outcomes: a systematic review and practical considerations. Sports medicine 47.9 (2017): 1821-1845

This is a peer-reviewed, author accepted manuscript of the following research article: Nashandi, H. L., Reilly, J. J., \& Janssen, X. (2020). Public health surveillance of physical activity in adolescents and adults in Namibia: a cross-sectional validation of activity questionnaires against accelerometry. Journal of Public Health. https://doi.org/10.1093/pubmed/fdaa067 